\title{
Revised statistics of radio halos and the reacceleration model
}

\author{
R. Cassano ${ }^{1,2}$, G. Brunetti ${ }^{2}$, T. Venturi ${ }^{2}$, G. Setti ${ }^{1,2}$, D. Dallacasa ${ }^{1,2}$, S. Giacintucci ${ }^{2}$, and S. Bardelli ${ }^{3}$ \\ 1 Dipartimento di Astronomia, Università di Bologna, via Ranzani 1, 40127 Bologna, Italy \\ e-mail: rcassano@ira.inaf.it \\ 2 INAF - Istituto di Radioastronomia, via P. Gobetti 101, 40129 Bologna, Italy \\ INAF - Osservatorio Astronomico di Bologna, via Ranzani 1, 40127 Bologna, Italy
}

Received 4 November 2007 / Accepted 18 December 2007

\begin{abstract}
Aims. The statistical properties of radio halos can be used to discriminate among the possible models for their origin. Therefore, an unbiased and exhaustive investigation of these properties is crucial.

Methods. With this goal in mind, in this paper, we revise the occurrence of radio halos in the redshift range $0-0.4$, combining the low redshift $(z<0.2)$ statistical study of the X-ray-brightest Abell-type clusters (XBACs) with the NRAO VLA Sky Survey (NVSS, by Giovannini et al. 1999) with our recent results from the radio followup of the ROSAT-ESO Flux-Limited X-Ray (REFLEX) clusters and extended ROSAT Brightest Cluster Sample (eBCS), i.e., the Giant Metrewave Radio Telescope (GMRT) radio halo survey, at higher redshift $(0.2<z<0.4)$.

Results. We find significant statistical evidence (at $3.7 \sigma$ ) of an increase of the fraction of clusters with radio halos with the X-ray luminosity (mass) of the parent clusters, and show that this increase is in line with statistical calculations based on the reacceleration scenario. We argue that a fundamental expectation of this scenario is that the probability of having radio halos emitting at hundred $\mathrm{MHz}$ is larger than the probability at $\mathrm{GHz}$ frequencies, and thus future radio interferometers operating at low frequencies, such as the Low Frequency Array (LOFAR) and the Long Wavelength Array (LWA), should detect a larger number of radio halos with respect to those caught by present $\mathrm{GHz}$ observations. We also show that the expected increase of the fraction of clusters with radio halos with the cluster mass as measured with future LOFAR and LWA surveys should be less strong than the increase in present surveys.
\end{abstract}

Key words. radiation mechanism: non-thermal - galaxies: clusters: general - radio continuum: general - X-rays: general

\section{Introduction}

Radio halos (RH elsewhere) are diffuse Mpc-scale radio sources observed at the center of a fraction of massive galaxy clusters (e.g. Feretti 2005, for a review). These sources are synchrotron emission from $\mathrm{GeV}$ electrons diffusing through $\mu \mathrm{G}$ magnetic fields and provide the most important evidence of nonthermal components in the intra cluster medium (ICM).

The clusters hosting RHs are always characterized by a peculiar dynamical status indicative of recent or ongoing merger events (e.g., Buote 2001; Schuecker et al. 2001), and this suggests a connection between the mergers and the origin of nonthermal components in the ICM. The main difficulty in understanding the origin of the synchrotron emitting electrons in RHs is that the lifetime of these electrons is much shorter than the diffusion time necessary to cover the scale of RHs. Two main possibilities have been explored to explain RHs: $i$ ) the secondary electron models, whereby the relativistic electrons are secondary products of the hadronic interactions of cosmic rays $(\mathrm{CR})$ with the ICM (e.g., Dennison 1980; Blasi \& Colafrancesco 1999), and $i$ ) the so-called reacceleration models, whereby relativistic electrons injected in the ICM are re-energized in situ by various mechanisms associated with the turbulence generated by massive merger events (e.g., Brunetti et al. 2001; Petrosian et al. 2001).

The two scenarios have different expectations for the basic statistical properties of RHs. In particular, in the secondary electron models, RHs should be long-living phenomena. Since the CR proton component is expected to be accumulated in clusters, the radio emission from secondary particles should be dominated at any time by the pile up of CRs protons during the merger history of the cluster, rather than by the latest merger event. On the contrary, in the reacceleration models RHs should be transient phenomena with relatively short lifetimes (1 Gyr or less) because of the finite dissipation timescale of the turbulence. The recent discovery that galaxy clusters with similar X-ray luminosity have a clear bimodal distribution in radio properties with only a fraction of these clusters hosting a RH (Brunetti et al. 2007) is in line with this latter scenario.

Particle reacceleration also provides a natural way to explain the morphology and spectral properties of the few well studied RHs and to account for the link between RHs and cluster mergers (e.g. Petrosian 2003; Brunetti 2004; Blasi 2004; Feretti 2005, for reviews). The physics of collisionless turbulence and the process of stochastic particle reacceleration are complex and rather poorly understood, however, recent calculations have shown that there may be room for sufficient Alfvénic and magnetosonic acceleration of particles in the ICM during cluster mergers (Brunetti et al. 2004; Brunetti \& Lazarian 2007), thus providing some physical support to this scenario.

Recent calculations in the framework of the reacceleration scenario have modelled the connection between RHs and cluster mergers in a cosmological framework, and derived the expected fraction of clusters with RHs as a function of the mass, redshift, and dynamical status of the clusters (Cassano \& Brunetti 2005, CB05; Cassano et al. 2006, CBS06). These calculations provide a unique predictive power and allow deep radio observations of samples of galaxy clusters to test the scenario and to constrain model parameters. 
Observational studies of the statistical properties of RHs can be very effective in constraining the origin of RHs and, in general, of the nonthermal components in galaxy clusters. Giovannini et al. (1999, GTF99) derived the occurrence of RHs in the X-ray-brightest Abell-type clusters (XBACs) by inspection of the NRAO VLA Sky Survey (NVSS, Condon et al. 1998) at $1.4 \mathrm{GHz}$; Kempner \& Sarazin (2001) carried out a similar study on the cluster sample selected by Abell et al. (1989, hearafter ACO sample) by means of the Westerbork Northern Sky Survey (WENSS, Rengelink et al. 1997) at $327 \mathrm{MHz}$. Both cluster samples are complete up to $z=0.2$. These studies show that RHs are rare at the detection level of the radio surveys used and that their detection rate increases with increasing the X-ray luminosity of the parent clusters: $30-35 \%$ for clusters with X-ray luminosity larger than $10^{45} h_{50}^{-1} \mathrm{erg} / \mathrm{s}$ were found to host RHs (GTF99).

Despite these observational claims, it is not clear whether the rarity of RHs and the increase of their occurrence with cluster mass is real or driven by selection biases due to the brightness limit of the NVSS and WENSS surveys (e.g., Rudnick et al. 2006).

In this paper, we extend these analyses up to larger redshift, $z \lesssim 0.4$. Most important, we derive unbiased statistics of RHs as we discuss the relevant radio and X-ray selection effects and limit our analysis to a cluster subsample that is not subject to these effects. The starting sample used is this paper is the combination of the NVSS-XBAC sample at $z<0.2$, and the recent sample of 50 massive (X-ray luminous) clusters at $0.2 \leq z \leq 0.4$ (Venturi et al. 2007) observed at $610 \mathrm{MHz}$ with the Giant Metrewave Radio Telescope (GMRT). The sample is presented in Sect. 2 and the selection effects are discussed in Sect. 3. In Sect. 4, we give the results on the occurrence of RHs, and in Sect. 5 they are compared with expectations from the reacceleration scenario. Finally, in Sect. 6 we discuss the main implications of this model for future low-frequency radio surveys. A $\Lambda \mathrm{CDM}$ $\left(H_{0}=70 \mathrm{~km} \mathrm{~s}^{-1} \mathrm{Mpc}^{-1}, \Omega_{\mathrm{m}}=0.3, \Omega_{\Lambda}=0.7\right)$ cosmology is adopted throughout.

\section{Selection of the cluster samples}

In this section, we describe the sample of galaxy clusters in the redshift bin $0-0.4$ from which we start our statistical analysis for the presence of RH. It consists of two X-ray selected cluster samples, at low and at high redshifts, with already available radio followups for the search of RH. Being X-ray selected, the sample may contain cooling core (CC) clusters. Because all RH are found in merging clusters, it is well known that a possible anticorrelation exists between the presence of CCs and of giant RHs at the cluster center (Edge et al. 1992; Feretti 2000). However, since our main aim is to perform an unbiased analysis of the occurrence of RH in galaxy clusters independent of their dynamical status, we will consider all clusters regardless of the presence of CCs.

\subsection{The sample at $z \leq 0.2$}

Following GTF99 at low redshifts, $z \leq 0.2$, we use the XBACs cluster sample (Ebeling et al. 1996). The XBACs is a complete, all sky X-ray flux limited sample of 242 clusters, which are optically selected from the catalogs of Abell (1958) and Abell et al. (1989, hearafter ACO), and compiled from the ROSAT All-Sky Survey (RASS). The sample is limited to high galactic latitude $\left(|b| \geq 20^{\circ}\right)$ and is statistically complete for X-ray fluxes larger than $5.0 \times 10^{-12} \mathrm{erg} \mathrm{s}^{-1} \mathrm{~cm}^{-2}$ in the $0.1-2.4 \mathrm{keV}$ band up to a redshift 0.2 , the nominal completeness limit of the ACO clusters (Ebeling et al. 1996), but there are also 24 clusters at redshift larger than 0.2 that meet the flux criterion.

This sample was cross-checked with the NVSS to search for RHs (GTF99). The NVSS is a radio survey performed at 1.4 GHz with the Very Large Array (VLA) in configurations $D$ and $D n C$. It has an angular resolution of $45^{\prime \prime}$ (HPBW), a surface brightness $\mathrm{rms}=0.45 \mathrm{mJy} /$ beam $(1 \sigma)$ and covers all the sky north of $\delta=-40^{\circ}$. Given the short baseline sampling available at the VLA, the NVSS is insensitive to diffuse sources on scales $\geq 15^{\prime}$, and this precludes the possibility to detect RH at $z<0.044$ (assuming $1 \mathrm{Mpc}$ size, GTF99). Thus, as a starting low-redshift subsample, we extract from the XBACs sample all clusters with $0.044 \leq z \leq 0.2$ and with $\delta>-40$. The final sample consists of 182 clusters (excluding A 1773 and A 388, which fall in the few remaining gaps of the NVSS).

\subsection{The sample in the redshift range: $0.2<z<0.4$}

The sample at larger redshift, $0.2<z<0.4$, consists of the combination of two X-ray subsamples with a relatively deep radio followup to search for $\mathrm{RH}$ recently performed at $610 \mathrm{MHz}$ with the GMRT. The two X-ray samples are the ROSAT-ESO Flux Limited X-ray (REFLEX) galaxy cluster catalog (Böringher et al. 2004) and the extended ROSAT Brightest Cluster Sample (eBCS) catalog (Ebeling et al. 1998, 2000). These two catalogs have almost the same flux limit in the $0.1-2.4 \mathrm{keV}$ band $\left(\gtrsim 3 \times 10^{-12} \mathrm{erg} \mathrm{s}^{-1} \mathrm{~cm}^{-2}\right)$ and their combination yields a homogeneous flux limited sample of clusters. GMRT radio followup of these catalogues has been performed by our group only for clusters with $L_{X}(0.1-2.4 \mathrm{keV}) \geq 5 \times 10^{44} \mathrm{erg} \mathrm{s}^{-1}$ extracted from the catalogues mentioned above. A description of the two resulting radio-X-ray samples is given below.

\subsubsection{The REFLEX subsample}

The REFLEX survey covers the southern sky up to a declination $\delta=+2.5^{\circ}$, avoiding the Milky Way and the regions of the Magellanic clouds, for a total area of $13924 \mathrm{deg}^{2}(4.24 \mathrm{sr})$. The sample is complete for $\mathrm{X}$-ray fluxes larger than $\sim 3 \times$ $10^{-12} \mathrm{erg} \mathrm{s}^{-1} \mathrm{~cm}^{-2}$ up to $z \sim 0.3$; above this redshift only very luminous objects (with X-ray luminosities of several $10^{45} \mathrm{erg} / \mathrm{s}$ ) have been observed (Böringher et al. 2001). To have a good $u-v$ coverage for the radio observations at the GMRT in this sample we selected only clusters with $\delta \geq-30^{\circ}$ (Venturi et al. 2007).

In Fig. 1, we report the distribution of the REFLEX clusters in the plane $L_{X}-z$, and with red circles we highlight the clusters matching our luminosity and declination criteria. We obtain a total sample of 27 clusters. The source list is reported in Table 1, where we give (1) the REFLEX name; (2) alternative name from other catalogs; (3) and (4) J2000 coordinates; (5) redshift; (6) the $\mathrm{X}$-ray luminosity in the $0.1-2.4 \mathrm{keV}$ band in unit of $10^{44} \mathrm{erg} \mathrm{s}^{-1}$; and (7) information on the diffuse emission.

Among these 27 clusters, three are clusters known to host RHs, i.e., A 2744, A 1300, and A 2163. From the remaining 24 clusters in Table 1 we selected all clusters with no radio information available in the literature and we also excluded those belonging to the GMRT Cluster Key Project (P.I. Kulkarni). The remaining 18 clusters (marked with the symbol $\sqrt{ }$ in Table 1 ) were observed with the GMRT (in several observational runs from January 2005 to August 2006). The total REFLEX sample with radio followup thus consists of 20 galaxy clusters 
Table 1. Cluster sample from the REFLEX catalog.

\begin{tabular}{|c|c|c|c|c|c|c|}
\hline REFLEX name & Alt. name & $\mathrm{RA}_{J 2000}$ & $\operatorname{Dec}_{J 2000}$ & $z$ & $\begin{array}{c}L_{\mathrm{X}} \\
10^{44} \mathrm{erg} / \mathrm{s}\end{array}$ & Diffuse emission \\
\hline$\sqrt{ }$ RXCJ 0003.1-0605 & A 2697 & 000311.8 & -060510 & 0.2320 & 6.876 & none \\
\hline ^ RXCJ 0014.3-3023 & A 2744 & 001418.8 & -302300 & 0.3066 & 12.916 & RH \\
\hline$\sqrt{ }$ RXCJ 0043.4-2037 & A 2813 & 004324.4 & -203717 & 0.2924 & 7.615 & none \\
\hline$\sqrt{ }$ RXCJ 0105.5-2439 & A 141 & 010534.8 & -243917 & 0.2300 & 5.762 & none \\
\hline$\sqrt{ }$ RXCJ 0118.1-2658 & A 2895 & 011811.1 & -265823 & 0.2275 & 5.559 & none \\
\hline$\sqrt{ }$ RXCJ 0131.8-1336 & A 209 & 013153.0 & -133634 & 0.2060 & 6.289 & RH \\
\hline$\sqrt{ }$ RXCJ 0307.0-2840 & A 3088 & 030704.1 & -284014 & 0.2537 & 6.953 & none \\
\hline${ }^{\circ} \mathrm{RXCJ} 0437.1+0043$ & - & 043710.1 & +004338 & 0.2842 & 8.989 & none \\
\hline$\sqrt{ }$ RXCJ 0454.1-1014 & A 521 & 045409.1 & -101419 & 0.2475 & 8.178 & relic \\
\hline RXCJ 0510.7-0801 & - & 051044.7 & -080106 & 0.2195 & 8.551 & $?$ \\
\hline$\sqrt{ }$ RXCJ 1023.8-2715 & A 3444 & 102350.8 & -271531 & 0.2542 & 13.760 & core halo \\
\hline$\sqrt{ }$ RXCJ $1115.8+0129$ & - & 111554.0 & +012944 & 0.3499 & 13.579 & none \\
\hline ^ RXCJ 1131.9-1955 & A 1300 & 113156.3 & -195537 & 0.3075 & 13.968 & RH \\
\hline RXCJ 1212.3-1816 & - & 121218.9 & -181643 & 0.2690 & 6.197 & $?$ \\
\hline$\sqrt{ }$ RXCJ 1314.4-2515 & - & 131428.0 & -251541 & 0.2439 & 10.943 & 2 relics, 1 small $\mathrm{RH}$ \\
\hline$\sqrt{ }$ RXCJ 1459.4-1811 & S 780 & 145929.3 & -181113 & 0.2357 & 15.531 & none \\
\hline RXCJ 1504.1-0248 & - & 150407.7 & -024818 & 0.2153 & 28.073 & $?$ \\
\hline$\sqrt{ }$ RXCJ 1512.2-2254 & - & 151212.6 & -225459 & 0.3152 & 10.186 & none \\
\hline RXCJ 1514.9-1523 & - & 151458.0 & -152310 & 0.2226 & 7.160 & $?$ \\
\hline${ }^{\star}$ RXCJ 1615.7-0608 & A 2163 & 161546.9 & -060845 & 0.2030 & 23.170 & RH \\
\hline$\sqrt{ }$ RXCJ $2003.5-2323$ & - & 200330.4 & -232305 & 0.3171 & 9.248 & RH \\
\hline RXCJ 2211.7-0350 & - & 221143.4 & -035007 & 0.2700 & 7.418 & $?$ \\
\hline$\sqrt{ }$ RXCJ 2248.5-1606 & A 2485 & 224832.9 & -160623 & 0.2472 & 5.100 & none \\
\hline$\sqrt{ }$ RXCJ 2308.3-0211 & A 2537 & 230823.2 & -021131 & 0.2966 & 10.174 & none \\
\hline$\sqrt{ }$ RXCJ $2337.6+0016$ & A 2631 & 233740.6 & +001636 & 0.2779 & 7.571 & none \\
\hline$\sqrt{ }$ RXCJ 2341.2-0901 & A 2645 & 234116.8 & -090139 & 0.2510 & 5.789 & none \\
\hline$\sqrt{ }$ RXCJ 2351.6-2605 & A 2667 & 235140.7 & -260501 & 0.2264 & 13.651 & none \\
\hline
\end{tabular}

Symbols are as follows: $\vee$ marks the clusters observed by us with the GMRT as part of our radio halo survey (Venturi et al. 2007; Venturi et al., in prep.); ${ }^{\star}$ marks the clusters with extended radio emission known from the literature (A 2744 Govoni et al. 2001; A 1300 Reid et al. 1999; A 2163 Herbig \& Birkinshaw 1994; and Feretti et al. 2001); ○ marks the clusters without extended radio emission known from the literature (RXCJ0437.1+0043 Feretti et al. 2005). The unmarked 5 clusters are part of the GMRT cluster Key Project (P.I. Kulkarni).

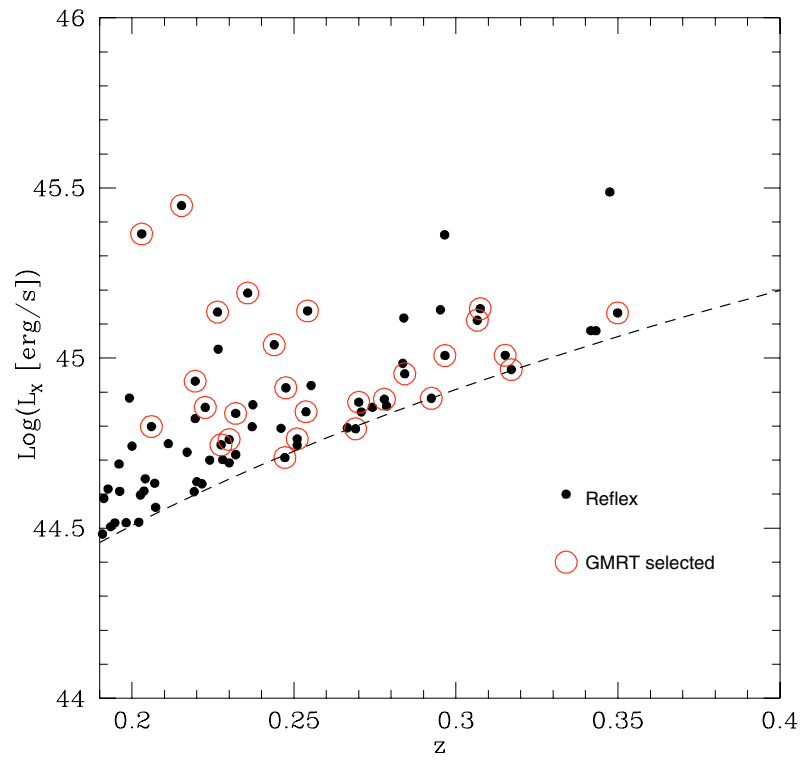

Fig. 1. X-ray luminosity $(0.1-2.4 \mathrm{keV})$ versus $z$ for the REFLEX clusters (black filled circles). Open red circles indicate the clusters belonging to our sample.

(here we exclude from this sample A 2163 and A 209, which are already included in the XBACs sample, and the clusters: RXCJ 0510.7-0801, RXCJ 1212.3-1816, RXCJ 1504.1-0248,
RXCJ 1514.9-1523, and RXCJ 2211.7-0350, which belong to the GMRT Cluster Key Project and still have no published data $\left.^{1}\right)$.

\subsubsection{The extended BCS subsample}

The ROSAT Brightest Cluster Sample (BCS; Ebeling et al. 1998) is a 90 per cent flux-complete sample of the 201 clusters of galaxies in the northen hemisphere selected from the RASS. All these clusters have flux higher than $4.4 \times 10^{-12} \mathrm{erg} \mathrm{cm}^{-2} \mathrm{~s}^{-1}$ in the $0.1-2.4 \mathrm{keV}$ band. This sample is combined with a lowflux extension of the BCS (Ebeling et al. 2000), which consists of 99 clusters of galaxies with flux higher than $2.8 \times$ $10^{-12} \mathrm{erg} \mathrm{cm}^{-2} \mathrm{~s}^{-1}$ in the $0.1-2.4 \mathrm{keV}$ band. The combination of these two samples forms the homogeneously selected extended BCS (eBCS), which is statistically complete up to a redshift $z \sim 0.3$ (Ebeling et al. 1998, 2000).

From the eBCS catalog we selected all clusters with $15^{\circ}<$ $\delta<60^{\circ}$, and obtained a total sample of 23 clusters (Venturi et al., in prep). In Fig. 2, we report the distribution of the eBCS clusters in the plane $L_{X}-z$, and with red circles we highlight the clusters that meet our selection criteria ${ }^{2}$.

\footnotetext{
1 The exclusion of these clusters from our sample does not introduce any bias because they are randomly distributed in redshift and X-ray luminosity among the clusters in the sample.

${ }^{2}$ Formally the eBCS subsample also contains RXCJ2228.6+2037, which is at $z>0.4$ and is not included in the following analysis.
} 
Table 2. Cluster sample from the eBCS catalog at $z<0.4$.

\begin{tabular}{|c|c|c|c|c|c|}
\hline Name & $\mathrm{RA}_{J 2000}$ & $\operatorname{Dec}_{J 2000}$ & $z$ & $\begin{array}{c}L_{\mathrm{X}} \\
10^{44} \mathrm{erg} / \mathrm{s}\end{array}$ & Diffuse emission \\
\hline$\sqrt{ }$ RXJ0027.6+2616 & 002749.8 & +261626 & 0.3649 & 12.29 & none \\
\hline${ } \mathrm{A} 611$ & 080058.1 & +360441 & 0.2880 & 8.855 & none \\
\hline$\vee_{A}$ 697 & 084253.3 & +362012 & 0.2820 & 10.57 & RH \\
\hline$\sqrt{ } Z 2089$ & 090045.9 & +205513 & 0.2347 & 6.79 & none \\
\hline${ }^{\star} \mathrm{A} 773$ & 091759.4 & +514223 & 0.2170 & 8.097 & RH \\
\hline${ }$ A781 & 092023.2 & +302615 & 0.2984 & 11.29 & relic? \\
\hline$\sqrt{ } \mathrm{Z} 2701$ & 095255.3 & +515252 & 0.2140 & 6.59 & none \\
\hline$\sqrt{ } \mathrm{Z} 2661$ & 094957.0 & +170858 & 0.3825 & 17.79 & complex $^{1}$ \\
\hline$\vee_{\text {A963 }}$ & 101709.6 & +390100 & 0.2060 & 6.39 & none \\
\hline$\sqrt{ }_{\mathrm{A} 1423}$ & 115722.5 & +33 3918 & 0.2130 & 6.19 & none \\
\hline$\sqrt{ }$ Z5699 & 130600.4 & +263058 & 0.3063 & 8.96 & none \\
\hline${ } \mathrm{A} 1682$ & 130649.7 & +463259 & 0.2260 & 7.017 & complex $^{1}$ \\
\hline$\sqrt{ }$ Z5768 & 131131.5 & +220005 & 0.2660 & 7.465 & none \\
\hline${ }^{\star} \mathrm{A} 1758 \mathrm{a}$ & 133232.1 & +503037 & 0.2800 & 12.26 & RH \\
\hline A1763 & 133517.2 & +405958 & 0.2279 & 9.32 & none \\
\hline$\sqrt{ } \mathrm{Z} 7160$ & 145715.2 & +222030 & 0.2578 & 8.411 & mini halo \\
\hline$\sqrt{ } Z 7215$ & 150123.2 & +422106 & 0.2897 & 7.34 & none \\
\hline$\sqrt{ }_{\mathrm{RXJ}} 1532.9+3021$ & 153254.2 & +302111 & 0.3450 & 16.485 & none \\
\hline A2111 & 153938.3 & +342421 & 0.2290 & 6.83 & none \\
\hline${ }^{\star} \mathrm{A} 2219$ & 164021.1 & +464116 & 0.2281 & 12.73 & RH \\
\hline A2261 & 172228.3 & +320913 & 0.2240 & 11.31 & uncertain $^{2}$ \\
\hline${ }^{\circ} \mathrm{A} 2390$ & 215334.6 & +174011 & 0.2329 & 13.43 & mini halo \\
\hline
\end{tabular}

Symbols are as follows: $\sqrt{ }$ marks the clusters observed with the GMRT as part of our radio halo survey (Venturi et al. 2007; Venturi et al., in prep.); ${ }^{\star}$ marks the clusters with a RH known from the literature (A 773 Govoni et al. 2001; A 1758 Giovannini et al. 2006 ; A 2219 Bacchi et al. 2003); ${ }^{\circ}$ marks the clusters with "mini-halo" known from the literature (A 2390 Bacchi et al. 2003). For clusters A 2111, A 1763 and A 2261, we analyzed $1.4 \mathrm{GHz}$ VLA archive data (Venturi et al., in prep.). ${ }^{1}$ The presence of extended radio galaxies, coupled with positive residuals at the cluster centre, does not allow us to establish the presence of possible cluster diffuse emission (Venturi et al. in prep.). ${ }^{2}$ The presence of a RH at the cluster center is uncertain: only B and D-array VLA data are available and extended sources at the cluster center do not allow for a firm conclusion (see discussion in Venturi et al., in prep.).

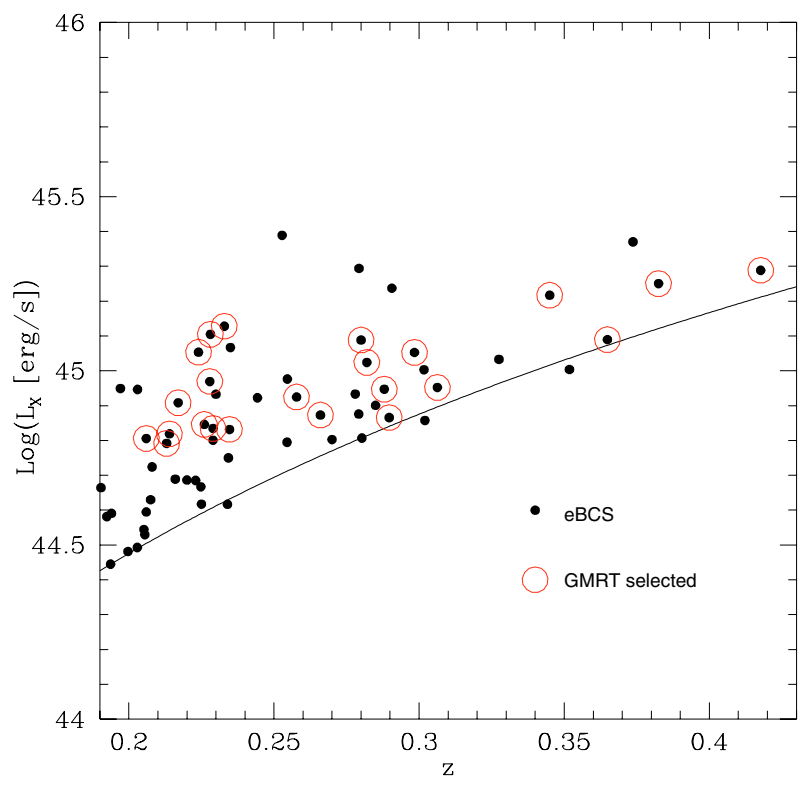

Fig. 2. X-ray luminosity $(0.1-2.4 \mathrm{keV})$ versus $z$ for the REFLEX clusters (black filled circles). Open red circles indicate the clusters belonging to our sample.

Table 2 gives details of the clusters extracted from the eBCS catalogue: (1) the cluster name; (2) and (3) J2000 coordinates; (4) redshift; (5) the X-ray luminosity in the $0.1-2.4 \mathrm{keV}$ band in unit of $10^{44} \mathrm{erg} / \mathrm{s}$; and (6) information on the diffuse emission. Among these 22 clusters $(z<0.4) 4$ have already known diffuse radio emission (A 773, A 1758, A 2219, A 2390), 15 were observed by our group at the GMRT (September 2005-August 2006), and for the remaining 3 clusters (A 2111, A 1763, and A 2261), we analyzed $1.4 \mathrm{GHz}$ data from archival deep pointed observations at the VLA (Venturi et al., in prep.).

The final X-ray selected sample with a radio followup at $z<$ 0.4 is thus made of 21 clusters (we also exclude A 963, which is already included in the XBACs sample).

\section{Radio selection effects}

The final sample of X-ray selected clusters with a radio followup from which we start our analysis is made by the combination of the XBAC-NVSS, the REFLEX-GMRT, and the eBCS-GMRT subsamples and consists of 220 galaxy clusters in the redshift interval $0.044<z<0.4$. The distribution of the clusters in the $L_{\mathrm{x}}-z$ plane is reported in Fig. 3, where we also highlight with red open circles the clusters with giant RH (GTF99; Venturi et al. 2007; Venturi et al., in prep.).

To perform a statistical census of the fraction of clusters with giant RHs in different ranges of X-ray luminosity and redshift, it is crucial to study the selection effects on the detection of RHs. These biases come from the strategy adopted in the radio followup of the different X-ray subsamples.

In the following, we discuss the selection effects on the NVSS and GMRT cluster samples and derive a X-ray subsample of clusters suitable for an unbiased statistical study of RHs. 


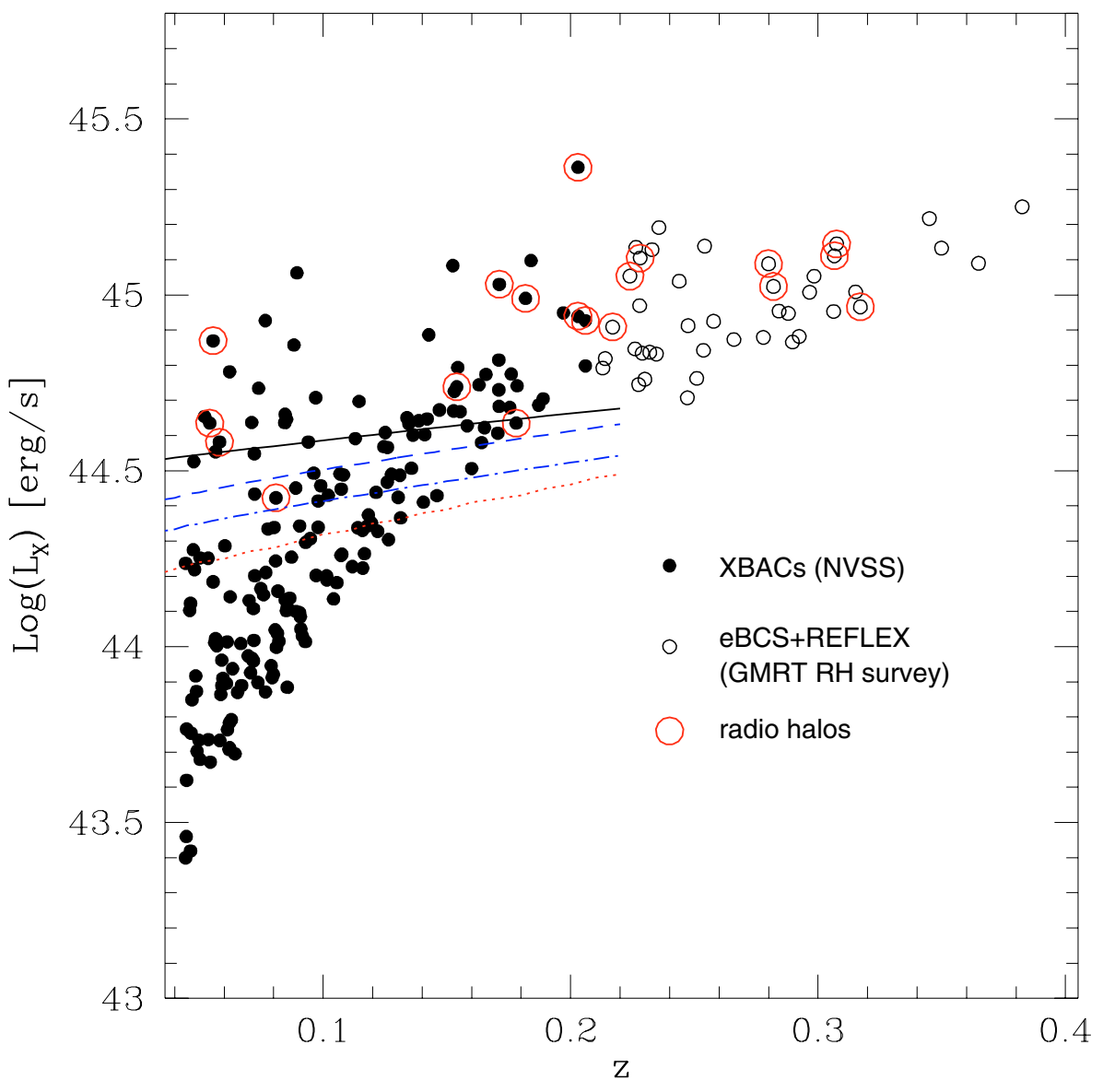

Fig. 3. X-ray luminosity $(0.1-2.4 \mathrm{keV})$ versus $z$ for the total sample of clusters (XBACs+REFLEX+eBCS). Open red circles indicate the clusters with known giant RHs. The lines give the lower limit to the cluster Xray luminosities in the case a) (black line), in the case b) (dotted line), and in the case c) (blue dot-dashed line: $2 \sigma$, blue dashed line: $3 \sigma$ ) as described in the text.

\subsection{The XBAC-NVSS subsample}

As previously reported, the NVSS is surface brightness-limited and RHs with a lower brightness are lost.

There is clear evidence of the existence of a correlation between the synchrotron radio power at $1.4 \mathrm{GHz}\left(P_{1.4}\right)$ of halos and the X-ray luminosity $\left(L_{\mathrm{X}}\right)$ of the parent clusters (Liang et al. 2000; Feretti 2000, 2003; Enßlin \& Röttgering 2002; Cassano et al. 2006). This correlation is not subjected to selection effects, at least for high X-ray luminosities $\left(\log \left(L_{X}\right)>44.6 \mathrm{erg} / \mathrm{s}\right.$; e.g., Clarke 2005; Dolag 2006; Brunetti et al. 2007) and it implies that the average brightness of RHs increases with the X-ray luminosity of the parent cluster (Feretti 2005; CBS06). If we scale the radio brightness with the cluster X-ray luminosity, the sensitivity limit in radio surveys will set the minimum surface brightness of a RH to be detected, and then a limiting X-ray luminosity of the hosting clusters.

An additional argument that should be used to discuss the selection of RHs in brightness limited surveys is their brightness distribution. In general, RHs are characterized by brightness profiles that smoothly decrease with distance from the cluster center (e.g., Govoni et al. 2001). The outermost low-brightness regions of RHs are lost in shallow surveys, however, what is important here, is the capability of these surveys to detect the central, brightest part of the diffuse emission. Indeed, the detection of these regions allows us to claim the presence of diffuse emission from clusters and to select samples of RH candidates for a deeper radio followup. By making use of several well-studied RHs, it was shown (Brunetti et al. 2007) that their integrated brightness profiles are quite similar, provided that their radial distance is normalised to the size of the different RHs. In particular, it has been shown that about half of the total radio flux density measured in RHs is contained in about half radius and this information can be used in discussing the selection of RHs in brightness limited surveys.

Thus, in discussing selection biases from the NVSS, we use both the $P_{1.4}-L_{\mathrm{X}}$ correlation and the constraints from the RH brightness profiles, and consider three possible approaches:

- case $a$ : we consider the case of RHs with a fixed radius, $R_{\mathrm{H}}=500 \mathrm{kpc}$. By making use of the $P_{1.4}-L_{\mathrm{X}}$ correlation, for each redshift we calculate the minimum $X$-ray luminosity of a cluster that can host RHs with an expected average radio brightness of $0.45 \mathrm{mJy} /$ beam within one half radius. The resulting minimum X-ray luminosity is reported as a function of redshift in Fig. 3 (solid black line);

- case b: we consider an additional correlation that has been found recently between the size of RHs and the synchrotron radio power at $1.4 \mathrm{GHz}, P_{1.4} \propto R_{\mathrm{H}}^{4.18 \pm 0.68}$ (Cassano et al. 2007, C07 hearafter): clusters with low X-ray luminosities should host smaller RHs than those with higher X-ray luminosities. This makes the detection of RHs in clusters with lower X-ray luminosity easier with respect to the previous case a. For each $z$, we compute the minimum radio power of RHs with an average radio brightness within one half radius (which depends on the radio power) of $0.45 \mathrm{mJy} / \mathrm{beam}$. The corresponding minimum X-ray luminosity of the clusters that may host these RHs is reported in Fig. 3 as a function of redshift (red dotted curve);

- case c: we consider RHs with fixed size, $R_{\mathrm{H}}=500 \mathrm{kpc}$, and with a typical brightness profile taken from Brunetti et al. (2007). For each redshift, we integrate the average brightness over an area as large as 4 times the NVSS beam and calculate the corresponding $1.4 \mathrm{GHz}$ power of RHs whose 

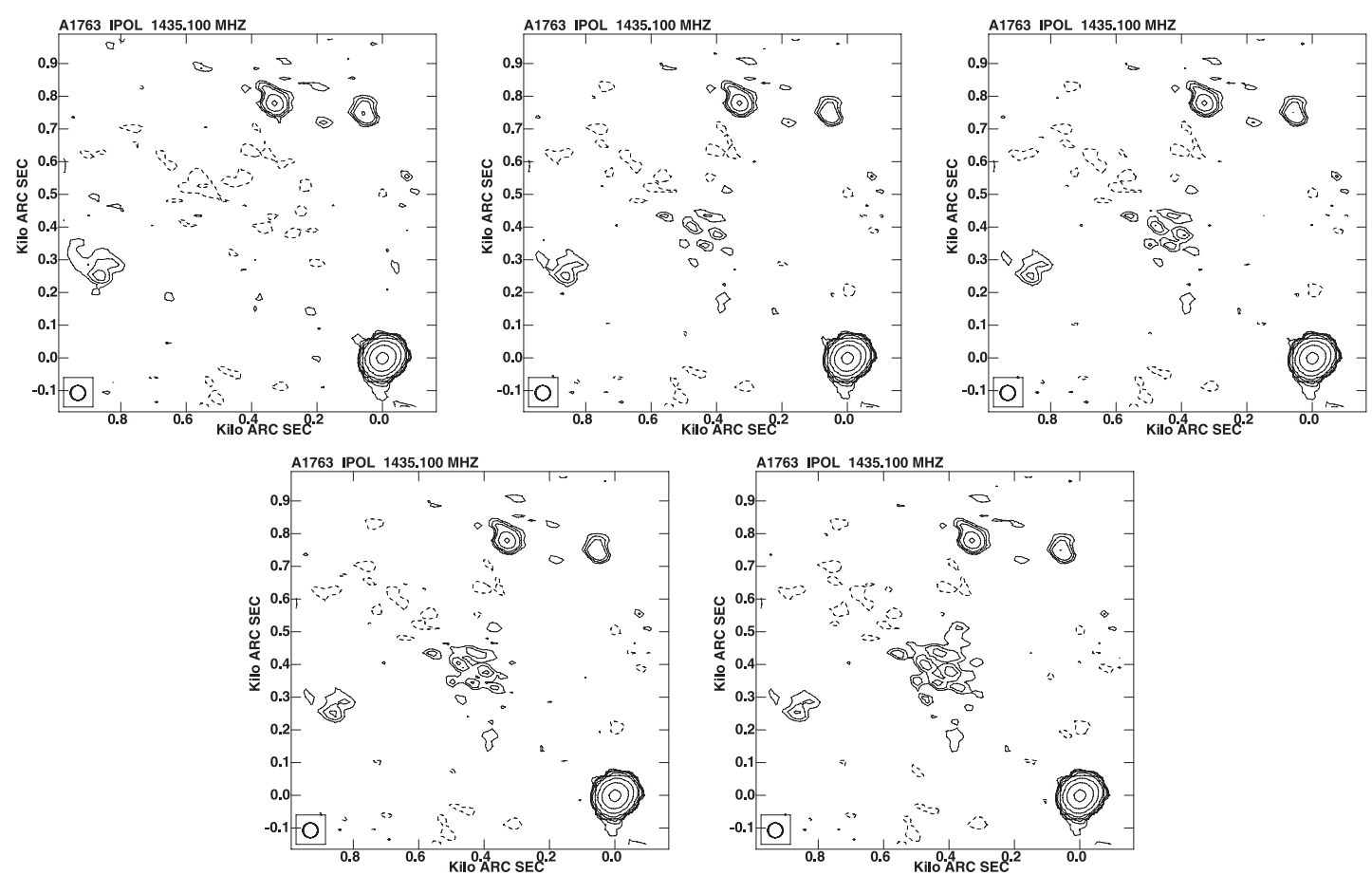

Fig. 4. Example of injection of fake RHs. We assume a size of $500 \mathrm{kpc}$ and a flux of 0,28, 32, 36 and $45 \mathrm{mJy}$ (from top left to bottom right panel). The rms in the images is of $0.45 \mathrm{mJy} / \mathrm{beam}$, the HPWB is $45^{\prime \prime} \times 45^{\prime \prime}$, contours are $1.0 \times(-1,1,1.4,2,4,8,32,125,500) \mathrm{mJy} / \mathrm{beam}$.

brightness equals 2 and 3 times the rms of the NVSS $(0.9$ and $1.35 \mathrm{mJy} /$ beam).

The corresponding minimum X-ray luminosity (from the $P_{1.4}-L_{\mathrm{X}}$ correlation) of clusters that may host these RHs is reported in Fig. 3 as a function of redshift (blue, dot-dashed and blue-dashed lines mark the case of 2 and 3 times the NVSS-rms, respectively).

In all the cases the NVSS is efficient in detecting RHs in clusters with $\log \left(L_{X}\right)>44.6 \mathrm{erg} / \mathrm{s}$. To strengthen the reliability of the above considerations on the efficiency of the NVSS in catching RHs, we use a more direct approach. Adopting the same procedure as Brunetti et al. (2007), we choose a cluster without $\mathrm{RH}$ and inject a "fake" $\mathrm{RH}$ in a region close to the pointing and without any significant contribution from real sources (upper left panel in Fig. 4). The "fake" RH was injected in the $u-v$ data by means of the task UVMOD in the software package AIPS and the resulting new data set was imaged using standard procedures. The brightness profile of the fake RH was modelled with a set of optically thin spheres with varying radius and flux density (Brunetti et al. 2007; Venturi et al., in prep). The $u-v$ data set used for this procedure was chosen from VLA archive observations carried out in the D configuration and it was edited so as to mimic a typical NVSS field in terms of $u-v$ coverage, sensitivity, and observing beam.

In Fig. 4, we report the results from the injection of fake RHs with fixed radius, $R_{\mathrm{H}}=500 \mathrm{kpc}$ and different total flux densities, and assuming $z=0.1$ (which is a mean value for the NVSS sample). From the left top panel to the right bottom panel, we report the results from injections of fake RHs with increasing total flux density. We find that diffuse emission is unambiguously imaged for RHs with flux density between 28 and $32 \mathrm{mJy}$. At the assumed redshift, this corresponds to $1.4 \mathrm{GHz}$ luminosity of RHs of $P_{1.4} \sim 7-8 \times 10^{23}$ Watt/Hz, which corresponds to cluster X-ray luminosity $L_{\mathrm{X}} \sim 4 \times 10^{44} \mathrm{erg} / \mathrm{s}$ (once the $P_{1.4}-L_{\mathrm{X}}$ is assumed), in good agreement with our estimates of the RHselection effects in the NVSS in the most conservative case a)
$\left(R_{\mathrm{H}}=500 \mathrm{kpc}\right.$, black line in Fig. 3), which therefore will be adopted in the following.

\subsection{The GMRT subsample}

The case of the GMRT subsample is different from that of the NVSS subsample, because the observations carried out by our group (Venturi et al. 2007; Venturi et al., in prep) are much deeper $\left(1 \sigma \sim 35-100 \mu \mathrm{Jy}\right.$ beam $\left.^{-1}\right)$ and aimed at the detection of RHs. This guarantees that the detection of extended diffuse emission at the level expected from the $P_{1.4}-L_{X}$ correlation is not biased by the sensitivity limit of those observations.

Brunetti et al. (2007) derived solid upper limits to the radio powers of clusters (in the GMRT sample) without evidence of diffuse radio emission. These upper limits lie about one order of magnitude below the RH luminosities expected on the basis of the $P_{1.4}-L_{\mathrm{X}}$ correlation. This allows us to conclude that the radio followup performed for the GMRT subsample does not introduce relevant biases to the detection of RHs in the cluster sample.

\section{Results: fraction of galaxy clusters with radio halos}

Based on the results reported in the previous sections, we define a new cluster sample that is made by the GMRT subsample (Sect. 2.2) and by all clusters of the XBACS-NVSS subsample (Sect. 2.1) not affected by biases in the detection of RHs, namely all those above the curves in Fig. 3.

In Fig. 3, the clusters hosting a giant RH are represented with open red circles: it is clear that RHs most likely occur in high $\mathrm{X}$-ray luminosity clusters, and this is consistent with previous claims (GTF99).

To obtain a statistically meaningful estimate of this "behaviour", we focus on the most conservative subsample, which is made by all clusters that lie above the upper curve in Fig. 3; 


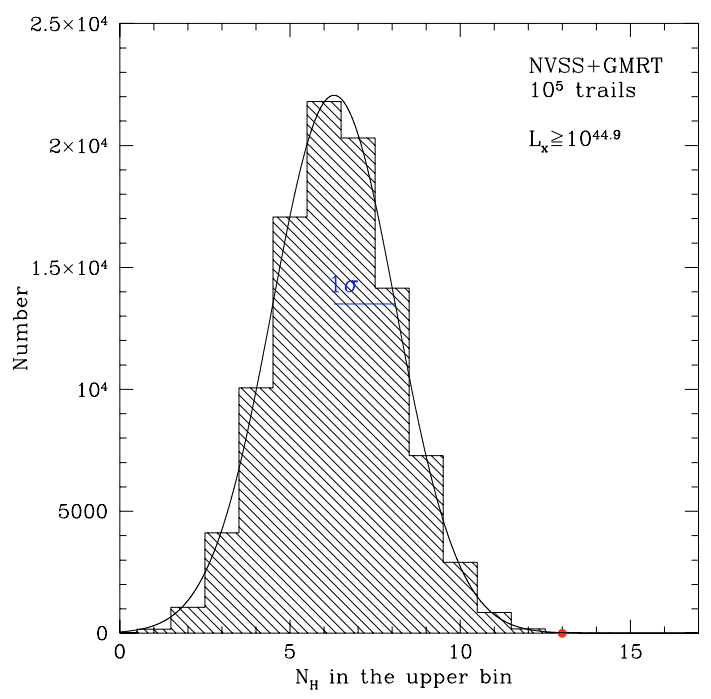

Fig. 5. Monte Carlo realization of $10^{5}$ trials in the HL clusters in the NVSS+GMRT sample.

this curve is derived by adopting the case a) (Sect. 3.1), which is supported by UVMOD simulations (Fig. 4).

As already anticipated the REFLEX and eBCS samples are complete at the X-ray flux limit up to $z \approx 0.3$, and Fig. 3 shows that incompleteness strongly affects the population of clusters with X-ray luminosity typical of the GMRT subsample at $z \geq 0.32$. Thus, we limit our analysis to the redshift range $0.044-0.32$ and calculate the fraction of clusters with RHs in the population of low luminosity (LL) and high luminosity (HL) clusters. We chose $L_{X}=10^{44.9} \mathrm{erg} / \mathrm{s}$ as the transition value between the LL and HL samples. Such value provides good statistics in both samples and ensures that the HL clusters cover the luminosity interval where the occurrence of RHs seems to increase (Fig. 3). The LL subsample is thus composed by those clusters with X-ray luminosity above the black curve in Fig. 3 and below $10^{44.9} \mathrm{erg} / \mathrm{s}$. We found that the fractions of clusters with RHs are $f_{\mathrm{RH}} \simeq 0.406 \pm 0.112$ and $f_{\mathrm{RH}} \simeq 0.075 \pm 0.038$ in the HL and LL subsamples, respectively ( $1 \sigma$ Poissonian errors).

This is the most conservative case. Indeed it is worth mentioning that by considering LL subsamples defined by other selection curves (case $\mathrm{b}$ and $\mathrm{c}$ ), smaller values of $f_{\mathrm{RH}}$ are obtained in the LL clusters: $f_{\mathrm{RH}} \simeq 0.063 \pm 0.026$ in the case b) (red dotted curve in Fig. 3$)$ and $f_{\mathrm{RH}} \simeq 0.074 \pm 0.030$ in the case c) $(2 \sigma$, blue dot-dashed line in Fig. 3).

In all these cases, there is clear evidence of an increase of the fraction of clusters with RHs with increasing the X-ray luminosity. To test the strength of this result we consider the most conservative case and run Monte Carlo calculations. Specifically, in this case the sample is composed by 85 clusters ( 32 are HL clusters and 53 are LL clusters) with 17 RHs, of which 13 are hosted by HL clusters and 4 by LL clusters. We randomly assign $17 \mathrm{RHs}$ among the 85 clusters of our sample and count the number of RHs that fall into the HL subsample in our Monte Carlo trials. In Fig. 5, we report the distribution of the number of RHs in the HL subsample obtained after $10^{5}$ Monte Carlo trials. The distribution can be fitted with a Gaussian function with a central value of $\sim 6.29$ and a standard deviation of $\sim 1.81$. This means that the observed value of $13 \mathrm{RHs}$ in the HL clusters is at $3.7 \sigma$ from the value expected in the case of $\mathrm{RH}$ occurrence independent of cluster X-ray luminosity, and thus that the probability to have the observed fraction of clusters with RHs by chance is $\leq 0.2 \times 10^{-3}$. Here we have assumed the presence of a $\mathrm{RH}$ in the cluster A 2261. This does not affect our result as the observed jump remains highly significant $(\sim 3.4 \sigma)$ even by assuming that A 2261 does not have a RH.

This shows that our result is statistically significant and suggests, for the first time, that there is a real (i.e., physical) increase of the occurrence of RHs with increasing the X-ray luminosity of the hosting clusters in an X-ray selected sample.

In principle, because we are using X-ray selected cluster samples, it is not clear whether the increase of the fraction of clusters with RHs across the LL and HL clusters is purely driven by the X-ray luminosity of clusters or is due to possible differences in the redshift distribution of clusters in the two HL and LL subsamples. In Fig. 6, we report the redshift distributions of clusters in the two subsamples. HL clusters have typically larger redshift because they can be detected by X-ray surveys at larger cosmic distance. More specifically, the mean redshift of LL clusters is $\sim 0.16$, while that of HL clusters is $\sim 0.23$. However, the difference in the redshift distribution is relatively small ( $\sim 0.74$ Gyr in cosmic time), and thus it is very unlikely that the increase (a factor of $\sim 5$ ) of the fraction of clusters with RHs observed in the two subsamples is due to some cosmic evolution of the population of RH sources. In addition, regardless of the origin of the particles emitting RHs, it should be mentioned that the radiative lifetime of these particles decreases with redshift due to inverse Compton (IC) losses and thus, in general, the fraction of clusters with RHs would be expected to decrease with cosmic look-back time. Finally, we note that the increase of the fraction of clusters with RH with cluster X-ray luminosity also appears considering the GMRT sample alone (Fig. 3); in this case clusters are essentially at the same redshift. Thus, although it is true that both luminosity and redshift may influence the occurrence of RHs (and model calculations that account for both effects are discussed in Sect. 5), we believe that the X-ray luminosity plays a major role in our cluster sample.

One possibility to further test this issue and to minimize the possible effect of the redshift on the observed fraction of clusters with RHs is to only consider the clusters of the XBACNVSS that lie above the most conservative radio selection-curve in Fig. 3; this sample is selected within $0.044<z<0.2$. In this case, however, it should be considered that the smaller cluster statistics and range of X-ray luminosities in the sample are expected to decrease the statistical significance of any possible trend observed for the fraction of RH clusters with cluster properties. Also, in this case, we derive the fraction of clusters with RHs in LL and HL clusters, but to maintain a sufficient cluster statistics in both the subsamples we adopt an X-ray luminosity threshold of $\sim 10^{44.8}$.

We find $f_{\mathrm{RH}} \simeq 0.43 \pm 0.17$ and $f_{\mathrm{RH}} \simeq 0.088 \pm 0.051$ in the HL and LL clusters, respectively, which again suggests an increasing fraction of clusters with RHs with increasing X-ray luminosity. As in the previous case, to test the statistical strength of these results, we perform Monte Carlo calculations by assuming an equal probability of having RHs in the clusters of the sample. In this case, we have a sample of 48 clusters (14 are HL clusters and 34 are LL clusters) with 9 RHs, of which 6 are in the HL subsample. In Fig. 7, we report the distribution of RHs in the HL subsample as counted after $10^{5}$ trials. The distribution is fitted with a Gaussian function that peaks at $\sim 2.49$ with a standard deviation of $\sim 1.29$, thus the observed value of 6 RHs is at $\sim 2.7 \sigma$ from that expected in the case of equal probability to have RHs with cluster X-ray luminosity; this means that the probability to obtain by chance the observed fraction of clusters with RHs is $\leq 9 \times 10^{-3}$. 


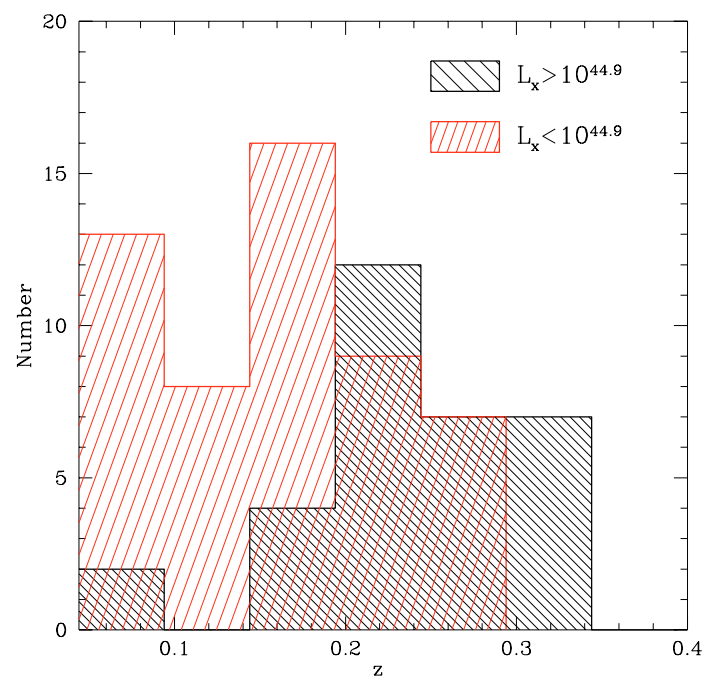

Fig. 6. Distribution of clusters as a function of redshift in the HL and LL subsamples.

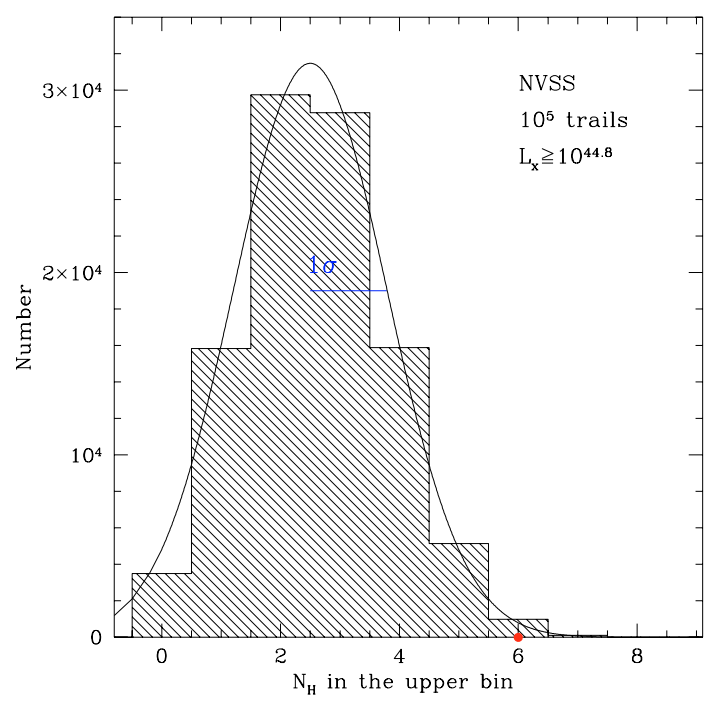

Fig. 7. Monte Carlo realization of $10^{5}$ trials for the number of RHs in HL clusters in the NVSS-XBACs sample.

Thus, although the significance of the result is reduced with respect to the case in which we consider the full NVSS+GMRT sample, the observed jump in the occurrence of RHs with the $\mathrm{X}$-ray luminosity is still statistically meaningful and further suggests that the effect is physically driven by cluster X-ray luminosity (or mass).

\subsection{The effect of cooling core clusters on the observed statistics}

It should be mentioned that the $P_{1.4}-L_{\mathrm{X}}$ correlation we used in Sect. 3 to derive the capability of the NVSS in catching RHs, is found for RH clusters that do not have a CC. In principle, the X-ray luminosity of these clusters should be corrected for the contribution of the $\mathrm{CC}$ when using the $P_{1.4}-L_{\mathrm{X}}$ correlation. Due to the lack of an adequate X-ray followup of the clusters in our sample, it is impossible to identify those affected by a CC. However, the fraction of CC clusters is found at a level of $~ 30 \%$ for BCS clusters with $L_{X} \geq 4 \times 10^{44}$ at $z \sim 0.15-0.4$ (Bauer et al. 2005), and this is unlikely to affect our main results. Indeed, once corrected from the CC contributions some HL clusters may become LL clusters and some LL clusters may fall below the Xray luminosity threshold and leave the selected sample. Because the LL and HL clusters are comparable in number, the occurrence of RH in both HL and LL clusters is expected to remain unchanged (although the fraction of RH in HL clusters may slightly increase). To better quantify this effect we performed Monte Carlo simulations. We assume that $30 \%$ of galaxy clusters of our sample are CCs and randomly assign CCs among clusters without RHs. Once the CC is assigned to a cluster we apply a correction to its X-ray luminosity, which is reduced by a factor randomly chosen between 1.4 and 2.5 (consistent with recent observations, e.g., Zhang et al. 2006, 2007; Chen et al. 2007), and re-evaluate the statistics of RH in the "corrected" LL and HL samples. In Fig. 8a, we report the distributions of the fractions of clusters with RH in the "corrected" sample, LL (left distribution) and HL (right distribution), obtained after having applied our procedure in $10^{6}$ Monte Carlo trials. The fractions of clusters with RHs in both LL and HL clusters only slightly increase with respect to the previous statistical calculation (Sect. 4). In Fig. 8b, we report the distribution of the ratio between the fraction of clusters with a RH in the "corrected" HL and LL samples obtained from $10^{6}$ Monte Carlo trials: it is clear that the majority of the values are consistent with those from the previous statistical analysis. These results, based on viable assumptions, show that the main finding of our work, the increase of the RH occurrence with the X-ray luminosity of galaxy clusters, might not be affected by the possible presence of CC clusters in the sample.

\section{A comparison between model expectations and observations}

In this section, we provide a basic comparison between present observations and the expectations of the reacceleration model in its simplest form. In the previous section, we showed that the fraction of clusters with RHs increases with the cluster X-ray luminosity and this provides an important piece of information that should be explained by the theoretical pictures for the origin of RHs. CB05 modelled the statistical properties of RHs in the framework of the merger-induced in situ particle acceleration scenario. By adopting the semi-analytic Press \& Schechter (1974) theory to follow the cosmic evolution and formation of a large synthetic population of galaxy clusters, we assumed that the energy injected in the form of magnetosonic waves during merging events in clusters is a fraction, $\eta_{t}$, of the $P \mathrm{~d} V$ work done by the infalling subclusters in passing through the most massive one. Then, the processes of stochastic acceleration of the relativistic electrons by these waves, and the ensuing synchrotron emission properties, have been calculated under the assumption of a constant magnetic field intensity averaged within a $1 \mathrm{Mpc}^{3}$ volume. CBS06 have extended this analysis by including a scaling of the magnetic field strength with cluster mass in the form $B \propto M_{\mathrm{v}}^{b}$. They showed that the observed correlations between the synchrotron radio power of a sample of $17 \mathrm{RHs}$ and the X-ray properties of the hosting clusters can be reproduced in the framework of the reacceleration model for $b \gtrsim 0.5$ and typical $\mu \mathrm{G}$ strengths of the average $B$ intensity. Those values provide a working framework in the range of $(B, b)$ model parameters under which the statistical properties of RHs, namely the occurrence of RHs with cluster mass and $z$, the luminosity functions and number counts, have been extensively calculated.

An important finding of this work was that generally the probability to form giant RHs increases with cluster mass, which 

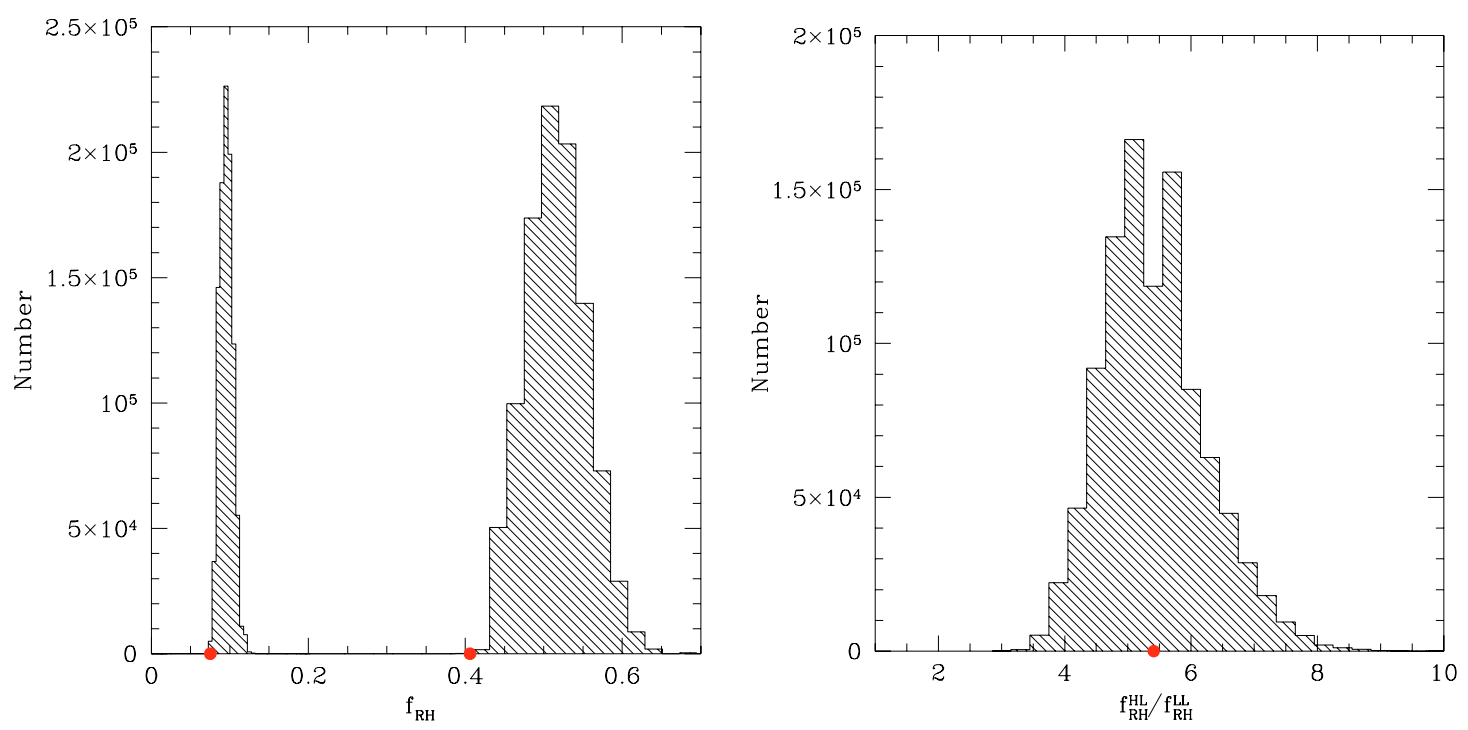

Fig. 8. a) Distributions of the fractions of clusters with RH in LL (left histogram) and HL (right histogram) subsample, obtained after $10^{6}$ Monte Carlo trials. The red filled circles rapresent the corresponding value of $f_{\mathrm{RH}}$ obtained without CC correction. b) Distribution of the ratio between the fraction of clusters with RH in HL and LL clusters obtained from $10^{6}$ Monte Carlo trials, the red filled circle being the value obtained without CC correction.

is a unique expectation of the reacceleration scenario. In particular, an abrupt increase of the fraction of clusters with RHs is expected across cluster masses of $\sim 2 \times 10^{15} M_{\odot}$, since the turbulent energy injected during cluster mergers scales with the cluster thermal energy (which roughly scales as $\sim M^{5 / 3}$ ), and turbulence in more massive clusters is injected on larger volumes (see CB05).

Specifically, the most important goal of this section is to test whether this reacceleration scenario can potentially match the increase of the fraction of clusters with RHs as derived from our cluster sample. We therefore adopt the same approach outlined in CB05 and CBS06 and compare model expectations with the results obtained in the previous section (case NVSS+GMRT samples).

To have a prompt comparison between model expectations and the data we calculate the expected fraction of clusters with RHs in different redshift bins by assuming the same redshift distribution of galaxy clusters as in Fig. 6. It should be mentioned that the model use virial masses $\left(M_{\mathrm{v}}\right)$ and thus to compare our expectations with observations we use the observed $L_{\mathrm{X}}-M_{\mathrm{v}}$ correlation (Eq. (7) in CBS06): $L_{\mathrm{X}} \propto M_{\mathrm{v}}^{1.47}$. This correlation is used to convert the range of X-ray luminosity of LL and HL clusters into mass ranges in which we compute the expected fraction of clusters with RHs.

In Fig. 9, we report the derived $f_{\mathrm{RH}}$ for different values of the model parameters (different lines) as a function of the X-ray luminosity overlaid on the observed values of $f_{\mathrm{RH}}$ (shadowed region). The model parameters are the magnetic field intensity, $B_{\langle M\rangle}$, of a cluster with virial mass $\langle M\rangle=1.6 \times 10^{15} M_{\odot}$ and the slope $b$ of the scaling of $B$ with $M_{\mathrm{v}}$ (see CBS06 for details). In all three cases, a value of $\eta_{t} \simeq 0.18$ is adopted: this value falls in the range of $\eta_{t}$ that has been shown to allow the reacceleration model, in the form developed by CB05 and CBS06, to be consistent with the production of RHs in $\approx 1 / 3$ of massive galaxy clusters. We find that the expected behaviour of the occurrence of RHs with cluster mass is consistent with the observed one for all the considered values of parameters.

It should be mentioned that the use of the $L_{\mathrm{X}}-M_{\mathrm{v}}$ correlation implies that $L_{X}$ reported in Fig. 9 is a good proxy for the mass. This $L_{\mathrm{X}}-M_{\mathrm{v}}$ correlation is obtained in CBS06 by using the

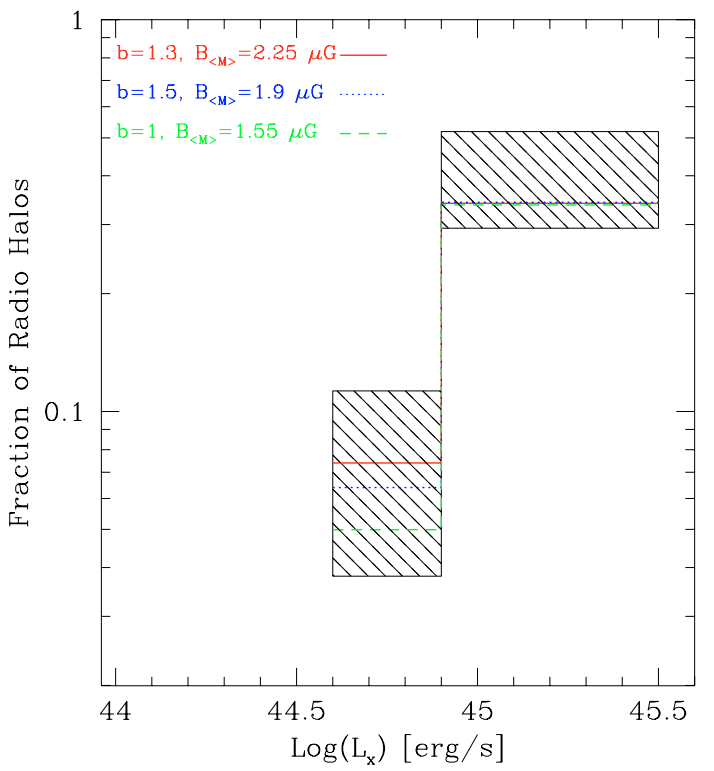

Fig. 9. Expected fraction of clusters with RHs in the HL and LL subsamples (different colored lines) vs the observed occurrence of RH with $1 \sigma$ errors (shadowed regions).

HIFLUGCS cluster sample (Reiprich \& Böhringer 2002), which contains $\mathrm{CC}$ and non-CC clusters and is characterized by a relatively large scatter in the mass, i.e. $\approx 45 \%$ (Reiprich \& Böhringer 2002). Very recent results on the Highest X-ray Flux Galaxy Cluster Sample (HIFLUGCS) showed that most of this scatter is due to the mixing of $\mathrm{CC}$ and non-CC clusters in the sample, with $\mathrm{CC}$ and non-CC clusters following correlations with compatible slopes (within $1 \sigma$ ), but different normalizations ${ }^{3}$ (Chen et al. 2007; Reiprich \& Hudson 2006).

Thus, the use of the $L_{\mathrm{X}}-M_{\mathrm{v}}$ correlation of CBS06 implies that the masses of non-CC clusters and $\mathrm{CC}$ clusters with a fixed $L_{\mathrm{X}}$ would be statistically underestimated and overestimated, respectively, and this would provide a compromise in the calculation of the masses spanned by the LL and HL clusters. A more

3 This is shown for both the $L_{\mathrm{X}}-M_{500}$ and $L_{\mathrm{X}}-M_{200}$ correlations. 

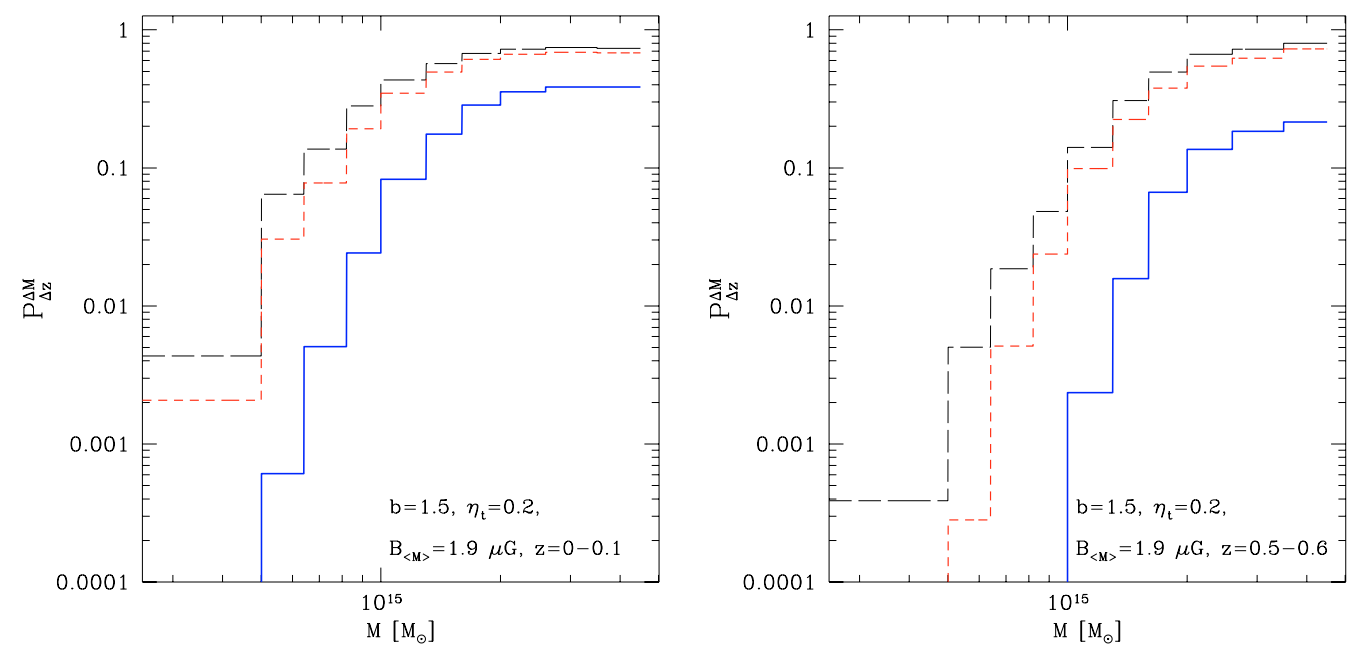

Fig. 10. Expected fraction of clusters with RHs at three different radio frequencies: $1.4 \mathrm{GHz}$ (blue-solid lines), $240 \mathrm{MHz}$ (red short-dashed lines) and $150 \mathrm{MHz}$ (black long-dashed lines) in the redshift bin $z \sim 0-0.1$ (left panel) and $z \sim 0.5-0.6$ (right panel). Calculations have been performed assuming: $b=1.5, B_{\langle M\rangle}=1.9 \mu \mathrm{G}$ and $\eta_{t}=0.2$.

detailed comparison would require the knowledge of the virial masses for all the clusters of our sample, which is however not available at the moment. Nevertheless this is unlikely to affect our results significantly. Indeed, one simple check is to use the $f_{\mathrm{RH}}$ in Fig. 8 (i.e., obtained from Monte Carlo simulations after statistical correction of the $L_{\mathrm{X}}$ of CC clusters) and to apply a systematic positive correction of $\approx 30 \%$ to the mass of LL and HL clusters with respect to the case of the $L_{\mathrm{X}}-M_{\mathrm{v}}$ correlation of $\mathrm{CBS} 6^{4}$. In this case we checked that the jump in $f_{\mathrm{RH}}$ and the values of $f_{\mathrm{RH}}$ themselves in Fig. 8 can be still well reconciled with the model expectations ${ }^{5}$.

\section{Radio halos in future low-frequency radio surveys}

In the previous section, we showed that the reacceleration scenario may explain the observed increase of the fraction of clusters with RHs with cluster X-ray luminosity (mass). On the other hand, from Fig. 9 it is evident that with the present surveys it is difficult to make a more quantitative statement, i.e., the poor statistics do not allow us to disentangle the different configurations of the model parameters.

In a few years, LOFAR and LWA will survey galaxy clusters with unprecedented sensitivity, thus increasing the observational statistics and hopefully providing a much clearer situation. On the other hand, these radio telescopes will observe at frequencies $\approx 10$ times lower than the present facilities and this could make the interpretation of the observations more tricky. Indeed, another natural expectation of the reacceleration scenario is that the fraction of clusters with RHs depends on the observing frequency. In particular, it is expected that the number of RHs should increase at low radio frequencies, $\approx 100 \mathrm{MHz}$, since low-energy electrons (with Lorentz factor $\gamma \approx 3000$ ) are able to radiate synchrotron emission at these frequencies and may also be reaccelerated in less energetic merger events (CBS06). For energetic reasons, it is also expected that this increase should be

\footnotetext{
${ }_{4}$ This correction has been estimated from the $L_{\mathrm{X}}-M_{200}$ correlation for non-CC clusters in Fig. 2 of Reiprich \& Hudson (2006).

5 We note that in this case, because the cluster masses (energetics) are slightly larger, the model formally reproduces $f_{\mathrm{RH}}$ for a slightly smaller value of $\eta_{t}, \eta_{t} \approx 0.16$.
}

more pronounced in the case of less massive clusters, which thus should host a sizable fraction of the population of RHs emitting at low radio frequencies.

The new generation of low-frequency interferometers, LOFAR and LWA, represents therefore a unique possibility to discover a new class of radio halos predicted on the basis of the reacceleration scenario that may emerge only at low radio frequencies. In particular, it is expected (CBS06) that the number of low-frequency RHs should be $\approx 10$ times that of "classical" $\mathrm{RHs}$ (those emitting at $\approx \mathrm{GHz}$ frequencies), thus the discovery of an excess of RHs at low frequencies with respect to the extrapolation of the number counts of present RHs would be a prompt confirmation of this scenario.

In Fig. 10, we report some preliminary calculations (based on CBS06, Sect. 7) of the probability to have RHs at different radio frequencies $(1.4 \mathrm{GHz}, 240 \mathrm{MHz}$, and $150 \mathrm{MHz}$, see figure caption). We found a large increase of the expected fraction of clusters with RHs at low radio frequencies (150-240 MHz); this increase is even more striking for less massive clusters, i.e., $M<10^{15} M_{\odot}$. Indeed, if the fraction of clusters with RHs in the redshift bin $0-0.1$ increases between $1400-150 \mathrm{MHz}$ by a factor of $\sim 2$ for $M \sim 2-3 \times 10^{15} M_{\odot}$, this increase is a factor of $\gtrsim 10$ for $M \lesssim 10^{15} M_{\odot}$. Furthermore, at higher redshifts, $z \sim 0.5-0.6$, this increase is even larger, a factor of $\sim 4$ and $\gtrsim 10^{2}$ in the case of $M \sim 2-3 \times 10^{15} M_{\odot}$ and $M \lesssim 10^{15} M_{\odot}$, respectively. From these results, we reach the important conclusion that the increase of the fraction of clusters with RHs with increasing cluster mass (or X-ray luminosity) should become less striking at low radio frequencies. In particular, considering the mass range in which present radio surveys have observed RHs, i.e., $10^{15} M_{\odot} \lesssim M \lesssim 4 \times 10^{15} M_{\odot}$, and considering two population of clusters with a separation mass $\sim 2 \times 10^{15} M_{\odot}$, the jump in the expected fraction is a factor of $\sim 4$ at $1.4 \mathrm{GHz}$ and is reduced to a factor of $\sim 2$ at $150 \mathrm{MHz}$. Thus, in general, LOFAR and LWA surveys are expected to find a less steep increase of the fraction of clusters with RHs as a function of the cluster mass with respect to present surveys.

\section{Conclusions}

The statistical properties of RHs are an important piece of information for the present understanding of non-thermal phenomena 
in galaxy clusters: do all clusters have a RH? Does the fraction of clusters with RHs depend on cluster mass and redshift?

In the past, a few seminal attempts were made to address these points (Giovannini et al. 1999; Kempner \& Sarazin 2001), however it was not clear how much those results were affected by the sensitivity limit of the adopted radio surveys. In particular, it was unclear whether the absence of RHs in low X-ray luminosity clusters could be ascribed to the steepness of the radio power-X-ray luminosity correlation of RHs combined with the sensitivity limit of the adopted surveys (Kempner \& Sarazin 2001).

In this work, we presented an unbiased statistical analysis of RHs in a large sample of clusters with radio information. We combined the subsample of XBACs clusters at $z \leq 0.2$, checked for the presence of diffuse radio emission in the NVSS by GTF99, with two subsamples of clusters in the redshift range 0.2-0.4 (extracted from the REFLEX and eBCS catalogs) inspected at $610 \mathrm{MHz}$ and at $1.4 \mathrm{GHz}$ with deep GMRT and VLA observations, the GMRT subsample (Venturi et al. 2007; Venturi et al., in prep.).

Brunetti et al. (2007) showed that the bulk of clusters of the GMRT sample does not show any hint of Mpc scale diffuse radio emission at their centres at the level of presently known RHs. For the clusters without extended radio emission, upper limits to their radio power were obtained: they are about one order of magnitude below the values expected on the basis of the radio power-X-ray luminosity correlation, and this allowed us to conclude that such correlation is real, i.e. not driven by observational biases at least for $L_{\mathrm{X}} \geq 5 \times 10^{44} \mathrm{erg} / \mathrm{s}$ (the lower X-ray luminosity of the GMRT sample).

Assuming that RHs follow this correlation, in this paper we have obtained the minimum X-ray luminosity of clusters that may host a RH detectable in the NVSS as a function of $z$. We followed a number of approaches based on the brightness profiles of well-studied RHs. In the most conservative case (case a, Sect. 3.1) we successfully test this approach by means of injection of fake RHs in a NVSS-like UV dataset. Based on these results for the NVSS and on those of Brunetti et al. (2007) for the GMRT sample, we were able to select a sample of clusters (NVSS+GMRT) that is not affected by observational biases and is suitable for a statistical analysis.

The main result of our analysis of this sample is that we find an increase of the fraction of clusters with RHs with the cluster $\mathrm{X}$-ray luminosity. More specifically, we find that the percentage of clusters at $z \approx 0.044-0.32$ with $4 \times 10^{44} \mathrm{erg} / \mathrm{s} \lesssim L_{X} \lesssim 8 \times$ $10^{44} \mathrm{erg} / \mathrm{s}$ hosting RHs is $7.5 \pm 3.8 \%$, while the percentage of clusters with $L_{X} \geq 8 \times 10^{44} \mathrm{erg} / \mathrm{s}$ hosting RHs is $40.6 \pm 11.2 \%$. We tested the significance of this result by means of Monte Carlo trials that allow us to conclude that the observed jump is real, with a significance of $3.7 \sigma$. In Sect. 4.1 we also showed that this result is not appreciably affected by the possible presence of $\mathrm{CC}$ clusters in the sample.

We showed that the increase of the fraction of clusters with RHs can be understood in the framework of the reacceleration scenario. This increase is a natural expectation of this scenario since a more efficient particle acceleration is triggered in massive (and X-ray luminous) clusters during merger events.

Since this scenario is at present in line with several observations, it is extremely intriguing, in view of forthcoming lowfrequency interferometers, such as LOFAR and LWA, to investigate the model expectations at low radio frequencies, i.e., a few hundreds MHz. In Sect. 6, we showed that this model predicts that the population of low frequency RHs is $\approx 10$ times larger than that of "classical" $\mathrm{RHs}$ (those emitting at $\approx \mathrm{GHz}$ frequencies), and we showed that the increase of the probability to have RHs emitting at low radio frequencies is maximized in the case of clusters with lower masses $\left(M \lesssim 10^{15} M_{\odot}\right)$. This implies that the increase of the fraction of clusters with RHs with cluster mass as measured by LOFAR and LWA surveys is expected to be less pronounced than that observed by present surveys.

Acknowledgements. This work is partially supported by MIUR, ASI, and INAF under grants PRIN2004, PRIN2005, PRIN-INAF2005, and ASI-INAF I/088/06/0. R.C. thanks F. Vazza for useful discussions. We acknowledge the anonymous referee for useful comments.

\section{References}

Abell, G. O. 1958, ApJS, 3, 211

Abell, G.O., Corwin, H. G. Jr., \& Olowin, R. P. 1989, ApJS, 70, 1

Bacchi, M., Feretti, L., Giovannini, G., \& Govoni, F. 2003, A\&A, 400, 465

Bauer, F. E., Fabian, A. C., Sanders, J. S., Allen, S. W., \& Johnstone, R. M. 2005, MNRAS, 359, 1481

Blasi, P. 2004, JKAS, 37, 483

Blasi, P., \& Colafrancesco, S. 1999, APh, 12, 169

Böhringer, H., Schuecker, P., Guzzo, L., et al. 2001, A\&A, 369, 826

Böhringer, H., Schuecker, P., Guzzo, L., et al. 2004, A\&A, 425, 367

Brunetti, G. 2004, JKAS, 37, 493

Brunetti, G., \& Lazarian, A. 2007, MNRAS, 378, 245

Brunetti, G., Setti, G., Feretti, L., \& Giovannini, G. 2001, MNRAS, 320, 365

Brunetti, G., Blasi, P., Cassano, R., \& Gabici, S. 2004, MNRAS, 350, 1174

Brunetti, G., Venturi, T., Dallacasa, D., et al. 2007, ApJ, 670, L5

Buote, D. A. 2001, ApJ, 553, 15

Cassano, R., \& Brunetti, G. 2005, MNRAS, 357, 1313 (CB05)

Cassano, R., Brunetti, G., \& Setti, G. 2006, MNRAS, 369, 1577 (CBS06)

Cassano, R., Brunetti, G., Setti, G., Govoni, F., \& Dolag, K. 2007, MNRAS, 378, 1565 (C07)

Clarke, T. E. 2005, ASP Conf. Ser. 345, ed. N. E. Kassim, M. R. Perez, W. Junor, \& P. A. Henning, 227

Condon, J. J., Cotton, W. D., Greisen, E. W., et al. 1998, AJ, 115, 1693

Dennison, B. 1980, ApJ, 239, L93

Dolag, K. 2006, Astron. Nachr., 327, 575

Ebeling, H., Voges, W., Bohringer, H., et al. 1996, MNRAS, 281, 799

Ebeling, H., Edge, A. C., Böhringer, H., et al. 1998, MNRAS, 301, 881

Ebeling, H., Edge, A. C., Allen, S. W., et al. 2000, MNRAS, 318, 333

Edge, A. C., Stewart, G. C., \& Fabian, A. C. 1992, MNRAS, 258, 177

Enßlin, T. A., \& Röttgering, H. 2002, A\&A, 396, 83

Feretti, L. 2000, Invited review at IAU 199, The Universe at Low Radio Frequencies, Pune, India, 1999

Feretti, L. 2003, in Matter and Energy in Clusters of Galaxis, ed. S. Bowyer, \& C.-Y. Hwang, ASP Conf. Ser., 301, 143

Feretti, L. 2005, in X-Ray and Radio Connections, published electronically by NRAO, ed. L. O. Sjouwerman, \& K. K. Dyer

Feretti, L., Fusco-Femiano, R., Giovannini, G., \& Govoni, F. 2001, A\&A, 373, 106

Feretti, L., Schuecker, P., Böhringer, H., Govoni, F., \& Giovannini, G. 2005, A\&A, 444, 157

Giovannini, G., Tordi, M., \& Feretti, L. 1999, NewA, 4, 141 (GTF99)

Giovannini, G., Feretti, L., Govoni, F., Murgia, M., \& Pizzo, R. 2006, Astron. Nachr., 327, 563

Govoni, F., Feretti, L., Giovannini, G., et al. 2001, A\&A, 376, 803

Herbig, T., \& Birkinshaw, M. 1994, BAAS, 26, 1403

Kempner, J. C., \& Sarazin, C. L. 2001, ApJ, 548, 639

Liang, H., Hunstead, R. W., Birkinshaw, M., \& Andreani, P. 2000, ApJ, 544, 686 Petrosian, V. 2001, ApJ, 557, 560

Petrosian, V. 2003, in Matter and Energy in Clusters of Galaxies, ed. S. Bowyer, \& C.-Y. Hwang (San Francisco: Astronomical Society of the Pacific), ASP Conf. Proc., 301, 337

Press, W. H., \& Schechter, P. 1974, ApJ, 187, 425

Reid, A. D., Hunstead, R. W., Lemonon, L., \& Pierre, M. M. 1999, MNRAS, 302,571

Rengelink, R. B., Tang, Y., de Bruyn, A. G., et al. 1997, A\&A, 124, 259

Rudnick, L., Delain, K. M., \& Lemmerman, J. A. 2006, Astron. Nach., 327, 549

Schuecker, P., Böhringer, H., Reiprich, T. H., \& Feretti, L. 2001, A\&A, 378, 408

Venturi, T., Giacintucci, S., Brunetti, G., et al. 2007, A\&A, 463, 937

Zhang, Y.-Y., Böhringer, H., Finoguenov, A., et al. 2006, A\&A, 456, 55

Zhang, Y.-Y., Finoguenov, A., Böhringer, H., et al. 2007, A\&A, 467, 437 